\title{
Eccentricity Based Topological Invariants of Tightest non-adjacently Configured Stable Pentagonal Structure of Carbon Nanocones
}

\author{
MIan Zobair ${ }^{1}$, Mehar Ali Malik², and Hani Shaker ${ }^{3}$ \\ ${ }^{1}$ National University of Sciences and Technology \\ ${ }^{2}$ Riphah International University - Lahore Campus \\ ${ }^{3}$ COMSATS Institute of Information Technology - Lahore Campus
}

June 18, 2021

\begin{abstract}
Conjugated open ended cones in which the configured pentagons are consistent, lies in the circle of Fries Kekule structure [8]. This non-adjacent tightest configuration of pentagons as shown in the Fig. 1 is consistent with a Fries Kekule structure and thus provides the most stable cone. In the study, various topological indices of the same structure in regard of physico-concoction resources and bioactivity of substance mixtures are studied which further helps to study the behavior of chemical compounds. In this regard, Zagreb indices $M_{-} 1^{\wedge *}(\mathrm{G})$ and $M_{-} 2^{\wedge *}(\mathrm{G})$ of a molecular graph G are used to evaluate the complexity in chemical systems and biological organisms. In this manuscript, we consider two complex families of stable carbon nanocones and compute their ECI, TEI and eccentricity-based Zagreb indices.
\end{abstract}

\section{Hosted file}

Eccentric-connectivity-index-2.pdf available at https://authorea.com/users/420361/articles/ 526725-eccentricity-based-topological-invariants-of-tightest-non-adjacently-configuredstable-pentagonal-structure-of-carbon-nanocones 